\title{
Engaging, not excluding: a response to Walsh et al.
}

\section{Elizabeth Pellicano, Ari Ne'eman and Marc Stears}

Although we warmly welcome efforts to outline the ethical challenges raised by the search for autism biomarkers, the recent Perspective article by Walsh et al. (In search of biomarkers for autism: scientific, social and ethical challenges. Nature Rev. Neurosci. 12, 603-612 $(2011))^{1}$ on this issue is confused. The authors are conscious of the need for "members of the autism community and their carers" to be involved in the biomarker research process, but the recommendations of their Perspective article preclude such engagement. Here, we highlight two troubling aspects of their argument and present an alternative.

First, despite the authors' interest in the wide range of responses to biomarker research, they do not acknowledge challenges to the research agenda itself. They thus overlook those who would prefer the emphasis of autism research to lie elsewhere, either for ethical or more practical reasons. Most straightforwardly, their Perspective article leaves the current flow of research resources unexamined. Had they analysed that flow, Walsh et al. would have noted that an overwhelming amount of current funding is granted for aetiological investigations, including biomarker research, with precious little being given to investigations aimed at improving the immediate quality of life of autistics and their families ${ }^{2}$. (Please note that we use the term 'autistics' rather than person-first language (for example, 'people with autism') as it is the preferred language of the Autistic Self Advocacy Network and of many people on the autism spectrum.) A comprehensive account of the challenges of biomarker research should have considered fully this opportunity cost.

Second, Walsh et al. note the intense disagreements regarding a 'prevention' agenda, one of the primary implications of autism biomarker research. They aspire to resolve ethical disputes concerning both the ethical acceptability of biomarker research and the impact such research is likely to have on health practice. Unfortunately, they suggest no mechanism by which scientists, autistics and the broader community might seek to overcome these disagreements collectively. Instead, when Walsh et al. turn to the root of these debates, they insist that "the final moral decision" lies with prospective parents, working together with genetic counsellors, without explaining why they consider these individuals to be the most appropriate people to resolve these disputes. Crucially, they suggest no means by which to also attend to the interests of autistics or their families.

We propose that three principles should guide ethically-informed autism biomarker research. First, scientists must acknowledge the continuing deep disagreements between involved parties regarding both the current research agenda and the consequential distribution of scarce resources. Second, they must recognize that the various parties, including autistics, their families and scientists, possess greatly varying degrees of power to shape the research process. Third, they must admit that all autism research, including basic science, biomarker and more applied work, has an impact on different groups in society autistics, carers, clinicians, researchers and the broader public - in profoundly different ways, and they must consider that impact throughout their research.

The only way to respond to these principles is for scientists to work immediately to create an open and participatory research process, as exemplified by community-based participatory research ${ }^{3}$. Although Walsh et al. helpfully remind us of the ethical and social dangers posed by biomarker research, they must accept that these dangers can only be overcome through the common endeavor of scientists, practitioners, autistics and their families, however challenging such engagement might be.

Elizabeth Pellicano is at the Centre for Research in Autism and Education, Department of Psychology and Human Development, Institute of Education, 25 Woburn Square, London WC1H OAA UK; and at the School of Psychology, University of Western Australia, 35 Stirling Highway, Crawley 6009, Australia.

Ari Ne'eman is at the Autistic Self Advocacy Network, Washington DC 20035 USA.

Marc Stears is at the Department of Politics and International Relations, University of Oxford, Manor Road, OXford OX1 3 UQ UK; and at the Institute for Public Policy Research, 14 Buckingham Street, London WC2N 6DF, UK.

Correspondence to E.P. e-mail: I.pellicano@ioe.ac.uk doi: 10.1038/nrn3113-c1

1. Walsh P., Elsabbagh, M., Bolton, P. \& Singh, I. In search of biomarkers for autism: scientific, social and ethical challenges. Nature Rev. Neurosci. 12, 603-612 (2011).

2. Singh, J., Illes, J., Lazzeroni, L. \& Hallmayer, J. Trends in US autism research funding. J. Autism Dev. Disord. 39, 788-795 (2009).

3. Nicolaidis, C., et al. Collaboration strategies in nontraditional community-based participatory research partnerships: lessons from an academic-community partnership with autistic self-advocates. Prog. Community Health Partnersh. 5, 143-150 (2011).

Competing interests statement

The authors declare no competing financial interests. 